

\title{
REFLECTANCIA DE LAS ENVOLVENTES VERTICALES Y SU INFLUENCIA SOBRE DISPONIBILIDAD DE LUZ NATURAL EN EL CAÑÓN URBANO DE LA CIUDAD DE CONCEPCIÓN.
}

\section{REFLECTANCE OF THE VERTICAL ENVELOPES AND ITS INFLUENCE ON THE AVAILABILITY OF NATURAL LIGHT IN THE URBAN CANYON OF THE CITY OF CONCEPCIÓN.}

\author{
SERGIO ANDRÉS VENEGAS QUINTULÉN \\ Magister Hábitat Sustentable y Eficiencia Energética \\ Universidad del Bío-Bío, Concepción Chile \\ svenegasq@gmail.com
}

\author{
MARÍA BEATRIZ PIDERIT MORENO \\ Departamento de Diseño y Teoria de la Arquitectura \\ Universidad del Bío-Bío, Concepción, Chile \\ mpiderit@ubiobio.cl
}

RESUMEN

El presente estudio tiene por objetivo evaluar la influencia de los materiales en las envolventes verticales sobre la disponibilidad de luz natural, en escenarios urbanos representativos de la ciudad de Concepción, con el fin de conocer el potencial de los materiales para aportar luz natural. La metodología se basa en la selección de casos de estudio del centro histórico de Concepción para armar modelos simplificados según su relación alto/ancho (H/W). Dentro de cada caso se escogen materiales de fachadas que muestren mayor presencia en el cañón urbano, para calcular

su reflectancia y ser aplicados a los escenarios simulados. Los resultados demuestran que la disponibilidad de luz natural exterior se ve directamente condicionada por la relación geométrica

del cañón urbano, la cual disminuye conforme aumenta la relación H/W. Se comprueba que el aporte lumínico debido al coeficiente de reflectancia del material que compone la fachada es mayor

conforme aumenta la relación H/W, es decir, mientras mayor sea la densidad del cañón urbano, mayor será la importancia de las propiedades reflectantes de los materiales de fachadas frente a la disponibilidad de luz natural.

\author{
Palabras clave \\ luz natural, reflectancia, cañón urbano, envolvente.
}

\begin{abstract}
The objective of this study is to evaluate the influence a vertical envelope's material has on the availability of natural light in urban settings representative of the city of Concepción, in order to know the potential of these materials to provide natural light. The methodology was based on the selection of case studies from the historic Concepción downtown to build simplified models according to their height/width ratio (H/W). In each case, façade materials with greater presence in the urban canyon were chosen to calculate their reflectance and use in the simulated settings. The results show that the availability of exterior natural light is directly conditioned by the geometric relationship of the urban canyon, which decreases as the H/W ratio increases. It was confirmed that the lighting contribution from the reflection coefficient of the façade's material is greater as the H/W ratio increases, that is to say, the greater the density of the urban canyon, the greater the importance of the reflective properties of the façade materials with regards to the availability of natural light.
\end{abstract}




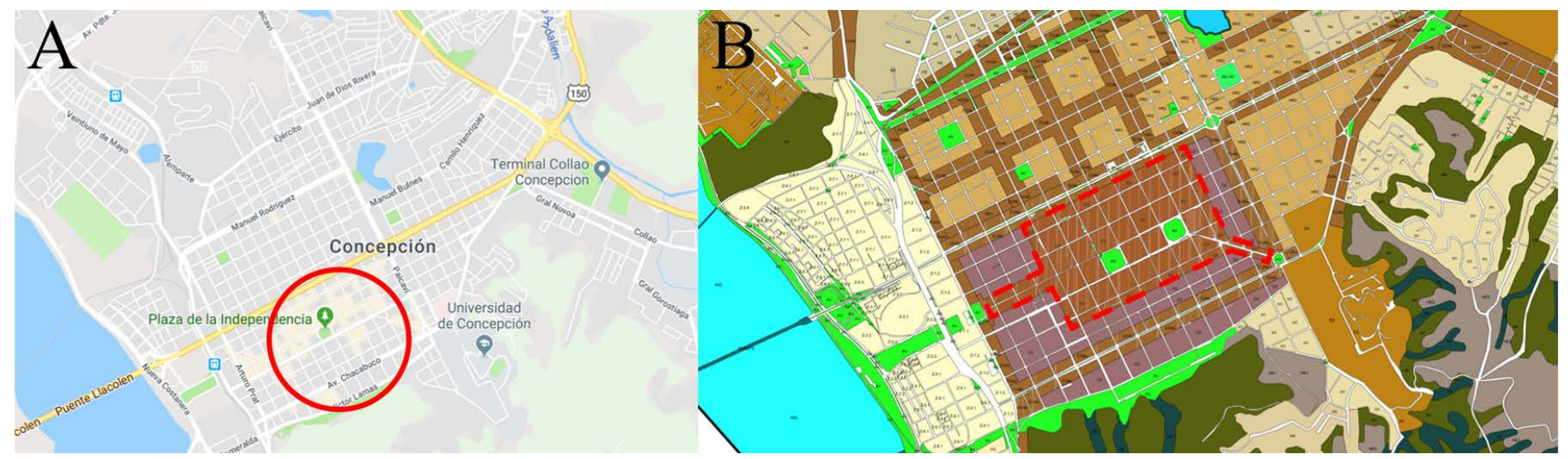

Figura 1: A: Plano de Concepción; B: Área de estudio.

Fuente: A: Google Maps; B: Plan Regulador Comunal de Concepción.

\section{INTRODUCCIÓN}

La superficie de la ciudad se compone de un mosaico de diferentes materiales, a los que denominamos la envolvente urbana (Alchapar y Correa, 2015). Cada uno de esos materiales muestra distintos comportamientos en función de sus propiedades ópticas, lo que impacta en la manera que percibimos el paisaje urbano. Así también, cada material que compone la envolvente urbana tiene diferente capacidad de reflejar la radiación solar recibida, lo cual altera la fracción de radiación absorbida o reflejada por la ciudad (ibídem). El concepto de reflectancia se refiere a una fracción de la radiación solar incidente reflejada de una superficie o un cuerpo (Gaffin et al., 2012), los que intervienen directa o indirectamente en la repartición y distribución de la luz dependiendo de su textura y su color (Ramírez y Orozco, 2015).

El vínculo entre densidad urbana y la disponibilidad de luz natural en los espacios habitables, ya sean exteriores o interiores, se encuentra en un complejo equilibrio entre factores propios del territorio y patrones espaciales del ambiente construido, el uso de los espacios urbanos, de los edificios y las propiedades ópticas de la envolvente urbana. El efecto de la sombra emitida por un edificio de gran altura es, en este contexto, un caso sencillo, ya que la incidencia de la sombra en otros edificios o espacios urbanos puede ser positiva o negativa, de acuerdo con las exigencias bioclimáticas del lugar y la estación (Mesa, Corica, y Pattini, 2011).

En términos geométricos, el cañón urbano se describe como la relación entre la altura de los edificios $(\mathrm{H})$ y el distanciamiento entre edificios adyacentes (W) (Strømann-Andersen y Sattrup, 2011). Pero, si bien el impacto de la geometría urbana y las cualidades reflectivas de los materiales en el microclima urbano está bien establecido (ibídem), los estudios han tendido a centrarse en problemas de sobrecalentamiento en climas cálidos, en el efecto de la isla de calor y en el confort urbano (Alchapar, Correa y Cantón, 2012; Alchapar y Correa, 2015; Yuan, Farnham y Emura, 2015). De esta manera, el impacto de los elementos arquitectónicos y reflejo de materiales fuera de los edificios no se ha estudiado en profundidad (Nasrollahi y Shokri, 2016).

La iluminación juega un papel fundamental para el desarrollo de las actividades sociales, educativas, comerciales e industriales
(Monteoliva y Pattini, 2013), pero la importancia de utilizar la luz natural dentro de los espacios habitables no radica sólo en el ahorro de energía, sino también en el hecho de que la mayoría de las personas muestran preferencia por la luz natural tanto en sus hogares como en edificios no residenciales (Mesa, Corica, y Pattini, 2011), por sus características ventajosas sobre la salud, el estado de ánimo y la productividad de las personas. Así como el empleo del tabique, el acero, la piedra y el concreto, la luz no debe emplearse como simple elemento decorativo sino como parte estructural de la arquitectura (Arias, Ávila, 2007).

El objetivo de este estudio es evaluar la influencia del coeficiente de reflectancia de materiales presentes en las envolventes verticales sobre la disponibilidad de luz natural, en escenarios urbanos representativos de la ciudad de Concepción, con el fin de conocer el potencial de dichos materiales en fachadas, para aportar luz natural aprovechable por los edificios.

La metodología utilizada para esos fines se basó, en una primera etapa, en la búsqueda y estudio de tipologías del cañón urbano en Concepción. Ello se desarrolló a través de un mapeo de la ciudad, haciendo uso de la Ordenanza Local del Plan Regulador Comunal de Concepción (Ilustre Municipalidad de Concepción, 2017), utilizando herramientas como Google Map y Google Earth y recorriendo las calles del centro de la ciudad para definir y contextualizar un área de estudio según la normativa y la realidad actual de la ciudad. La segunda etapa consistió en la selección de cañones urbanos proporcionados entre sí, dentro del área de estudio, para generar casos basados en su relación H/W y materialidad (Strømann-Andersen y Sattrup, 2011). Luego se escogieron las materialidades de fachadas más predominantes y se determinaron los valores de reflectancias ( $\rho$ ) para cada una de ellas. La reflectancia se determinó a través del método hemisphericalhemispherical reflectance $\left(\rho_{\text {hl }}\right)$ (Córica y Pattini, 2005), haciendo uso de un medidor de luminancia Konica Minolta LS-110 y una tarjeta de grises Vitamall utilizada en fotografía, como estándar para el balance de exposición y color. A partir de las muestras obtenidas, se seleccionaron tres materialidades representativas ( $\rho$ menor, $\rho$ promedio y $\rho$ mayor), lo que permitió conocer el aporte del coeficiente de reflectancia sobre la disponibilidad de luz natural exterior. En la tercera etapa, se analizaron los aportes de luz natural en las envolventes verticales de cada casos de 
cañón urbano mediante simulación dinámica, con el programa Radiance (a través SketchUp plugin Groundhog), considerando los coeficientes de reflectancia definidos, la orientación de las dos fachadas que componen el cañón y los distintos niveles de pisos. Radiance es un programa de simulación lumínica altamente capaz (Greenup y Edmonds, 2004) que ha sido validado por diferentes investigadores en diferentes casos y los resultados obtenidos se han encontrado en buen acuerdo con las mediciones experimentales y reales (Freewan, Gharaibeh y Jamhawi, 2014).

\section{DESARROLLO}

\section{ESTUDIO DE TIPOLOGÍAS MORFOLÓGICAS DEL CAÑÓN URBANO DE LA CIUDAD DE CONCEPCIÓN}

Se delimita como área de estudio al sector céntrico de la ciudad perteneciente a la Zona C1 del Plan Regulador Comunal de Concepción (Figura 1). Se escoge este sector debido a su condición céntrica y a que comprende la zona con la mayor densificación dentro del tejido urbano de Concepción. Por esta razón, la Zona C1 es un sector crítico, en donde la altura de las edificaciones y ancho de las vías juegan un rol primordial en la cantidad de luz natural captada por el espacio urbano y la influencia de la materialidad de la envolvente urbana será mayor.

Se definen las orientaciones predominantes dentro de la trama urbana de la ciudad de Concepción para ser aplicadas a los casos de cañones y estudiar escenarios en distintos contextos de asoleamiento. Las orientaciones se agrupan principalmente en noreste/suroeste y noroeste/sureste, cuyos ángulos, medidos en sentido de las agujas del reloj, entre el eje norte y el eje de la calzada, son de $62,76^{\circ}$ y $152,76^{\circ}$, y se encuentran enfrentando las fachadas noroeste/sureste y noreste/suroeste, respectivamente (Figura 2).

Para la selección de casos se otorgó prioridad a aquellas secciones del área de estudio que presentaran mayor continuidad a lo largo de sus fachadas, con el fin de acentuar la influencia de los materiales en la disponibilidad de luz natural. Se buscaron secciones cuyas alturas edilicias fueran relativamente constantes para simplificar el análisis y que no existieran grandes diferencias entre la realidad y el modelo de cañón urbano. Se determinó, asimismo, una dimensión estándar de tres metros por nivel con el propósito de establecer la altura total de cada edificio de manera aproximada.

Concepción posee una variedad de tipologías en fachadas y cada una de ellas con un porcentaje de acristalamiento diferente, por lo que se escogió una tipología que fuera representativa de la ciudad (Figura 3). Así, se seleccionó un porcentaje de acristalamiento de $25 \%$, el cual es común en los edificios históricos del centro de la ciudad.

Seguidamente, se analizaron las tipologías morfológicas presentes en el área de estudio para generar una muestra de casos diferenciados entre sí y de manera proporcionada entre sus relaciones $\mathrm{H} / \mathrm{W}$, en aras de asegurar una muestra
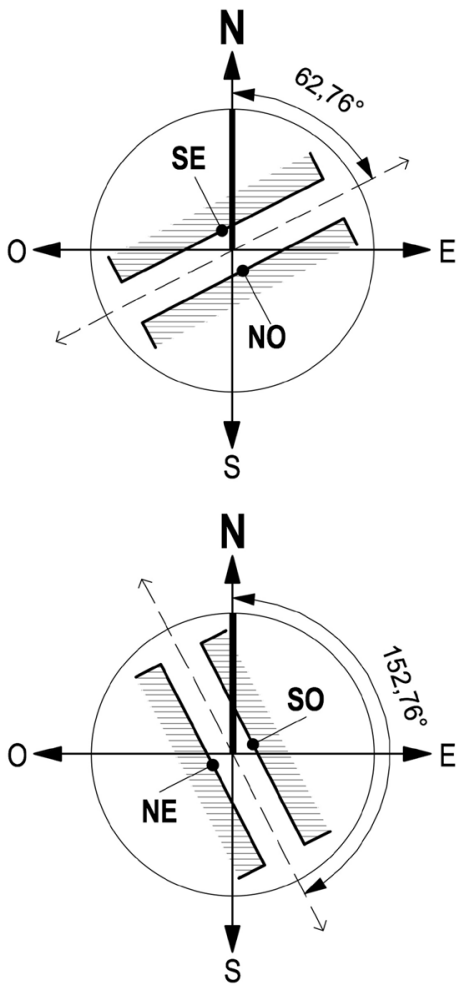

Figura 2: A: Orientación 1 (fachadas noroeste/sureste); B: Orientación 2 (fachadas noreste/suroeste). Fuente: Elaboración del autor.

variada y equilibrada. Además, se seleccionaron seis casos de cañones urbanos, tres por orientación, de tal forma que fueran representativos entre las mayores y menores relaciones H/W encontradas en el área de estudio. Se realizó el levantamiento del perfil de los seis cañones seleccionados a partir de sus orientaciones y relaciones $\mathrm{H} / \mathrm{W}$, calculadas en relación las alturas edilicias obtenidas y el distanciamiento entre las fachadas de cada caso (Figura 4).

Como es inexacta la altura promedio del perfil de los casos, en función de la dimensión estándar de tres metros, se modificó el último nivel, aumentando o disminuyendo la altura y la superficie de ventanas de éste, de modo que la altura del perfil total concordara con el promedio obtenido en cada caso y que la superficie de ventanas correspondiera al 25\%, según su análisis (Tabla 1).

\section{DEFINICIÓN MATERIALIDADES PREDOMINANTES.}

Se escogieron materiales de fachadas predominantes dentro de los casos seleccionados, para ser medidos conforme al método "hemispherical-hemispherical reflectance" $\left(\rho_{h h}\right)$. (Tabla 2). Se eligió como pavimento la superficie de hormigón ya que es el material más común dentro de los casos. El vidrio seleccionado es un vidrio simple cuyas propiedades ópticas son las establecidas en el programa Radiance. Estos materiales son constantes y se aplican a todos los modelos de cañones urbanos para igualar o asemejar las condiciones de análisis de todos los casos. 

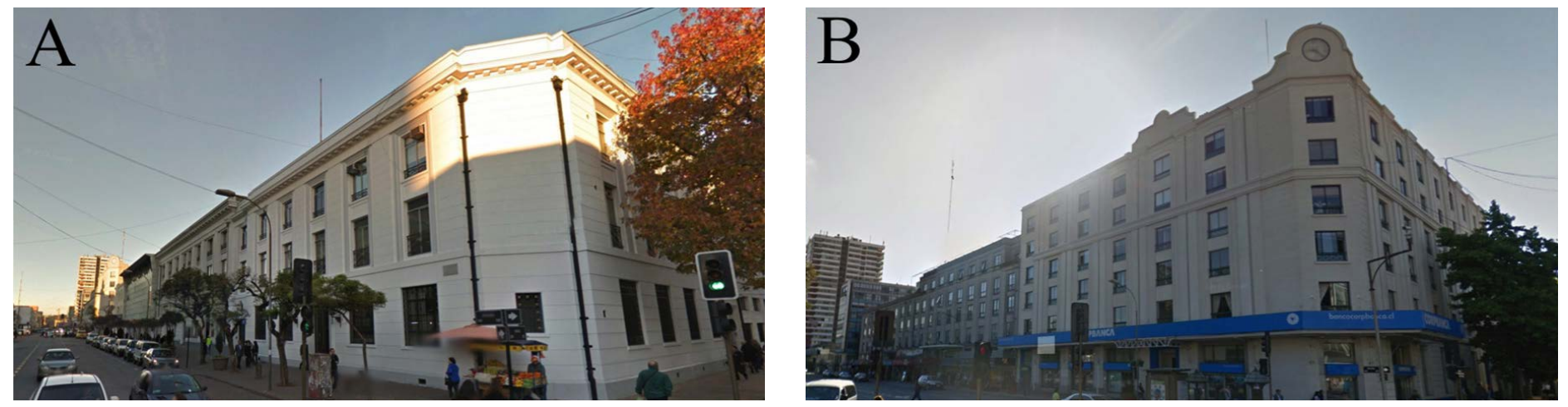

Figura 3: A: Edificio esquina O’Higgins/Caupolicán; B: Edificio esquina O'Higgins/Rengo. Fuente: Google Street View.

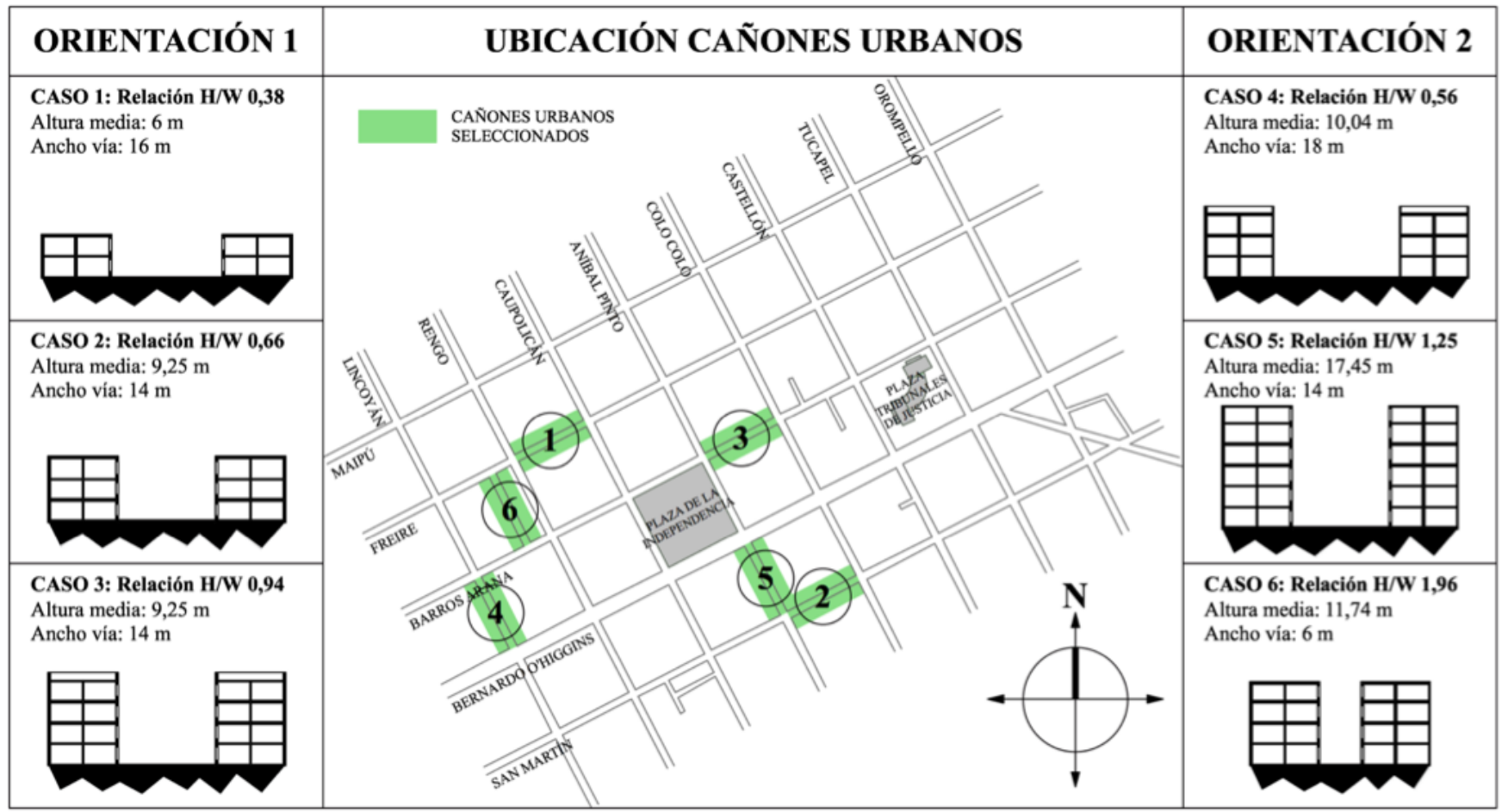

Figura 4: Perfil de cañones urbanos seleccionados y ubicaciones.

Fuente: Elaboración del autor.

\begin{tabular}{|c|c|c|c|c|c|}
\hline $\begin{array}{c}\text { Cañones } \\
\text { Urbanos }\end{array}$ & $\begin{array}{c}\text { Altura promedio } \\
(\mathbf{m})\end{array}$ & $\mathbf{N}^{\circ}$ niveles & Altura último nivel $(\mathbf{m})$ & Superficie Unidad (m2) & Superficie vano (m2) \\
\hline Caso 1 & 6,00 & 2 & 3,00 & 30,00 & 7,50 \\
\hline Caso 2 & 9,25 & 3 & 3,25 & 32,50 & 8,13 \\
\hline Caso 3 & 13,09 & 4 & 4,09 & 40,90 & 10,23 \\
\hline Caso 4 & 10,04 & 3 & 4,04 & 40,40 & 10,10 \\
\hline Caso 5 & 17,45 & 6 & 2,45 & 24,50 & 6,13 \\
\hline Caso 6 & 11,74 & 4 & 2,74 & 27,40 & 6,85 \\
\hline
\end{tabular}




\begin{tabular}{|c|c|c|c|c|c|c|c|c|c|}
\hline IMAGEN & MATERIAL & TERMINACIÓN & COLOR & $\begin{array}{l}\mathrm{cd} / \mathrm{m}^{2} \\
\text { tarjeta } \\
\text { blanca }\end{array}$ & $\begin{array}{c}\mathrm{cd} / \mathrm{m}^{2} \\
\text { tarjeta gris }\end{array}$ & $\begin{array}{c}\mathbf{c d} / \mathbf{m}^{2} \\
\text { material }\end{array}$ & p1 & р2 & phh \\
\hline & Hormigón & Rugoso, texturado & Gris & 575,2 & 115,4 & 139,6 & 0,22 & 0,22 & 0,22 \\
\hline & Cerámica & Liso, texturado & Ocre & 1380 & 263,7 & 525,4 & 0,34 & 0,36 & 0,35 \\
\hline & Hormigón & Liso & Beige & 1160 & 271 & 591,7 & 0,46 & 0,39 & 0,43 \\
\hline & Mortero & Rústico & Café & 1328 & 212,9 & 508,7 & 0,34 & 0,43 & 0,39 \\
\hline & Piedra pizarra & Texturado & Gris & 687,2 & 138,5 & 172,9 & 0,23 & 0,22 & 0,23 \\
\hline & Albañilería & Liso & Ocre & 1009 & 213,4 & 149,2 & 0,13 & 0,13 & 0,13 \\
\hline & Mortero & Rústico & Azul & 1065 & 231,2 & 115,1 & 0,10 & 0,09 & 0,09 \\
\hline & Mortero & Rústico & Blanco & 1233 & 239,1 & 1025 & 0,75 & 0,77 & 0,76 \\
\hline & Cerámica & Liso, texturado & Beige & 353,6 & 63,98 & 185,1 & 0,47 & 0,52 & 0,50 \\
\hline & $\begin{array}{l}\text { Granito } \\
\text { reconstituido }\end{array}$ & Rugoso, grano fino & Gris & 32,61 & 6,72 & 12,34 & 0,34 & 0,33 & 0,34 \\
\hline & Hormigón & Liso & $\begin{array}{c}\text { Naranjo } \\
\text { Pastel }\end{array}$ & 717 & 142 & 551,3 & 0,69 & 0,70 & 0,70 \\
\hline $8=n^{2}$ & $\begin{array}{l}\text { Granito } \\
\text { reconstituido }\end{array}$ & Liso, grano grueso & $\begin{array}{c}\text { Amarillo } \\
\text { pastel }\end{array}$ & 333,8 & 68,49 & 154,8 & 0,42 & 0,41 & 0,41 \\
\hline & Hormigón & Liso & Blanco & 141,6 & 31,14 & 138,6 & 0,88 & 0,80 & 0,84 \\
\hline $\begin{array}{l}\text { Reflectancia } \\
\text { promedio }\end{array}$ & & & & & & & & & 0,43 \\
\hline
\end{tabular}

Tabla 2: Muestra de materiales con cálculo de reflectancia según método "hemispherical-hemispherical reflectance" $\left(\rho_{\text {hh }}\right)$.

Fuente: Elaboración del autor.

El método hemispherical-hemispherical reflectance ( $\rho$ hh) consiste en la obtención de la reflectancia de un material a través de cálculos de proporción, con los que se obtienen dos reflectancias ( $\rho 1$ y $\rho 2$ ). El cálculo se lleva a cabo a través de un medidor de luminancia que permite conocer los valores de candela por metro cuadrado $\left(\mathrm{cd} / \mathrm{m}^{2}\right)$ tanto del material a analizar como de las tarjetas de color blanco $(\rho: 0,9)$ y gris medio $(\rho: 0,18)$, cuyas reflectancias son conocidas. De esta forma se realizan los cálculos de proporción en base a la tarjeta blanca $(\rho 1)$ y la tarjeta gris $(\rho 2)$, para ser promediados y el resultado obtenido correspondería a la reflectancia del material analizado ( $\rho h h)$.

\section{ESTUDIO DE LOS APORTES DE LUZ NATURAL EN ENVOLVENTE VERTICALES.}

\section{Parámetros de simulación}

Los cielos que con mayor frecuencia se presentan en Concepción, según la herramienta Ilumina Chile (Piderit), son el cielo intermedio (parcial nublado) junto al cielo cubierto (nublado); ambos se presentan con una frecuencia de un $32 \%$ en la ciudad. Para las simulaciones del estudio se seleccionó el cielo intermedio por presentar luz solar directa como variable, permitiendo escenarios diferentes dentro de un año calendario en cuanto a condiciones de intensidad, altura y ángulo solar. Los valores de iluminancia exterior simulados son los establecidos en el programa Radiance para cielos intermedios (Radiance Script: \#gensky mes día hora -a -36.77 -o $73.05-m+60+i>$ sky.rad).

El programa SketchUp se utilizó para modelar los casos de estudio por la sencillez en su utilización, su condición de gratuidad y por ser compatible con varias herramientas de análisis lumínico. Los escenarios de cañones urbanos se modelaron considerando la relación H/W de cada caso, un porcentaje de ventanas de $25 \%$ y la reflectancia de tres materiales para las fachadas (Tabla 2).

Para la modelación de los escenarios de cañones urbanos, se definió una unidad básica que corresponde a un recinto de 50 $\mathrm{m} 2$ cuyas dimensiones son $10 \mathrm{~m}$ de frente, $5 \mathrm{~m}$ de profundidad y $3 \mathrm{~m}$ de alto con un porcentaje de ventanas $25 \%$ en fachada, que corresponde a 3 ventanas de 1,4 m por 1,79 m (Figura 5). Se aplicaron grillas verticales en el área de las ventanas del módulo central de cada caso con el fin de conocer la disponibilidad de luz natural exterior aprovechable por los edificios en estos 


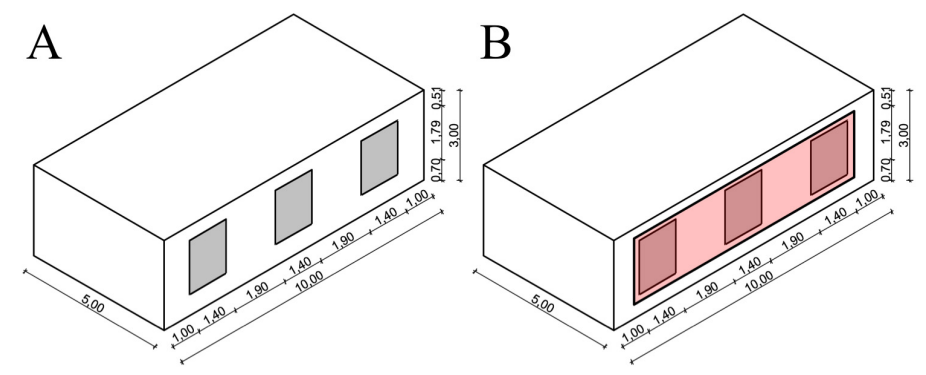

Figura 5: A: Unidad básica de cañones urbanos; B: Módulo central con grilla vertical.Fuente: Elaboración del autor.

puntos (Figura 6). Las grillas se aplicaron a través de una extensión gratuita y de código abierto de SketchUp llamada Groundhog (plugin), que permite crear rápidamente modelos que son leídos y procesados por el programa Radiance y así realizar el análisis de iluminación.

El escenario de cañón urbano se simuló considerando grillas verticales ubicadas en las fachadas de cada nivel de piso, a la altura de las ventanas, del módulo central y para ambas orientaciones predominantes: noroeste/sureste y noreste/ suroeste (Figura 6). Luego se realizaron simulaciones de iluminancia para conocer la disponibilidad de luz natural exterior en cada uno de los escenarios conforme a su relación H/W y la reflectancia del material de fachadas.

Para las simulaciones se utilizó una matriz de $3 \times 3$, considerando 3 fechas críticas (21 de junio, 21 de septiembre y 21 de diciembre) y 3 horas (9.00, 13.00 y 17.00 hrs.). La matriz $3 \times 3$ es una metodología que permite realizar estudios lumínicos y de asoleamiento en fechas claves dentro de un año calendario como son el solsticio de invierno, equinoccio y solsticio de verano, junto a tres unidades de tiempo representativas (mañana, medio día, y tarde), que definen un intervalo de tiempo perteneciente a un período normal de uso. Los resultados se promedian para obtener un valor único de iluminancia por cada uno de los niveles de piso y fachadas de cada caso con el fin de simplificar la investigación.

Los materiales simulados son el mortero proyectado azul ( $\rho 9$ $\%)$, el hormigón color beige ( $43 \%$ ) y el hormigón color blanco ( $\rho 84 \%$ ), los que dan cuenta de un coeficiente de reflectancia bajo, medio y uno alto, que permitirá conocer la influencia sobre la disponibilidad de luz natural que significa el uso de cada uno de ellos. Se realiza también un análisis comparativo porcentual de los materiales de acuerdo a la relación de sus reflectancias; para tal efecto, se seleccionó la relación $\rho 84$ $\%$ / $\rho 9 \%$ y $\rho 84 \%$ / $\rho 43 \%$ con el propósito de conocer el aporte de luz natural que se logra al aumentar el valor de la reflectancia de los materiales en fachadas, en los distintos contextos de cañones urbanos.

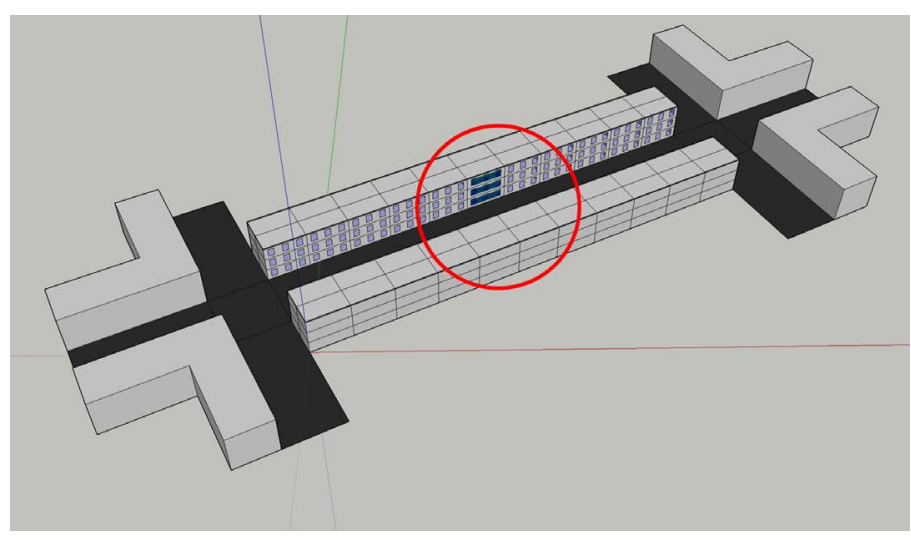

Figura 6: Ejemplo de caso de cañón urbano con grillas verticales en módulos centrales de cada fachada y nivel de piso. Fuente: Elaboración del autor.

\section{RESULTADOS DEL APORTE DE LUZ NATURAL}

Los casos se agrupan en las dos orientaciones predominantes de la trama urbana de la ciudad (noroeste/sureste y noreste/ suroeste). Los resultados analizados corresponden al promedio de los valores de iluminancia obtenidos en las simulaciones (según la matriz de $3 \times 3$ ), pertenecientes a ambas fachadas y los niveles de piso de cada cañón urbano, a partir de los cual es posible realizar un análisis comparativo entre los casos estudiados, de modo de conocer el aporte de luz natural de los materiales de fachadas en distintos contextos urbanos de manera simplificada.

\section{Orientación 1: fachadas noroeste/sureste}

La orientación 1 comprende los casos 1, 2 y 3 con relaciones H/W de 0,38, 0,66 y 0,94 respectivamente (Figura 4). Los resultados revelan diferencias de iluminancia entre las fachadas noroeste y sureste de entre $40 \%$ y 50 \% (Figura 7), siendo en el caso 1 donde esta diferencia es mayor, la cual es de valores cercanos a 6000 lux. Los mayores valores de iluminancia se obtienen en la fachada noroeste donde se alcanzan valores superiores a los 10000 lux mientras que los menores valores son cercanos a los 6000 lux, obtenidos en la fachada sureste. El caso 1, por su baja relación H/W, es el caso con los mayores valores de iluminancia, alcanzando valores cercanos a los 12000 lux en la fachada noroeste y sobre los 6000 lux en la fachada sureste, mientras que el caso 3, al poseer una mayor relación H/W, alcanza los menores valores de iluminancia, los que son de entre 8000 y 10000 lux en la fachada noroeste y de valores cercanos a 6000 lux en la fachada suroeste. En la Figura 7 se observa que las diferencias de iluminancia alcanzadas entre los distintos coeficientes de reflectancia son similares y regulares para todos los casos, las cuales son de valores de entre 1500 y 2000 lux, comparando las reflectancias de 9\% y $84 \%$. Se observan también diferencias de iluminancia entre los distintos niveles de piso en los casos conforme la relación H/W del cañón urbano, donde los primeros niveles alcanzaron los menores valores de iluminancia y los últimos niveles alcanzan los mayores valores para todos los casos. Los casos 1 y 2 exhiben valores de iluminancia relativamente semejantes entre 
los niveles de piso de los cañones urbanos, pero en el caso 3 , por su mayor relación $\mathrm{H} / \mathrm{W}$, se advierte una gran diferencia entre los niveles de la fachada noroeste, la cual es de 2000 lux comparando los niveles 1 y 4, mientras que en la fachada sureste los valores de iluminancia se mantienen más regulares.

En relación al porcentaje de aporte lumínico por reflectancia del material en fachadas, se muestra, en la Figura 7, que el aporte de luz natural por reflectancia del material es mayor conforme aumenta la diferencia de reflectancias comparadas. Se aprecia también que el aporte será mayor conforme aumenta la relación H/W, principalmente en la fachada sureste, donde se alcanzan aportes del $12 \%$ y $25 \%$ en el caso 1 (mientras que el caso 3 los aportes alcanzan los $20 \%$ y $40 \%$ ), comparando los coeficientes de reflectancia $84 \%$ / $43 \%$ y $84 \%$ / $9 \%$, respectivamente.

Según los niveles de piso de los cañones urbanos, se observa que los casos 1 y 2 no evidencian grandes diferencias de iluminancia entre sus niveles de piso por su baja relación H/W, pero en el caso 3, esta diferencia de iluminancia es más evidente debido a su mayor relación H/W. En la fachada noroeste, el porcentaje de aporte lumínico se mantiene relativamente constante entre los distintos niveles de piso, y en la fachada sureste se visualiza que en los primeros niveles el aporte de luz natural es mayor para todos los casos, principalmente en el caso 3 donde los aportes en el primer nivel alcanzan los 20 $\%$ y $40 \%$, mientras que en el cuarto nivel son de $15 \%$ y $30 \%$, comparando los valores de reflectancia $84 \%$ / $43 \%$ y $84 \%$ / 9\%, respectivamente.

Las diferencias de iluminancia entre niveles de piso y fachadas ocurren debido a que, en los casos de mayor relación H/W, los primeros niveles de piso reciben una mínima cantidad de luz solar directa; lo mismo sucede con la fachada sureste, por lo que la contribución de luz solar indirecta, a través de la reflectancia de los materiales, es mayor. Respecto a las fachadas, la sureste es la más favorecida, ya que las fachadas no se benefician de su propia luz reflejada, sino de la reflejada por las fachadas que se les enfrentan, es decir, la noroeste (válido para la orientación 2).

\section{Orientación 2: fachadas noreste/suroeste.}

La orientación 2 comprende los casos 4, 5 y 6, con relaciones H/W de 0,56, 1,25 y 1,96 respectivamente (Figura 4). Los resultados revelan diferencias de iluminancia entre las fachadas noroeste y sureste de entre $30 \%$ y $40 \%$ (Figura 8 ), lo que se traduce en una diferencia aproximada de 4000 lux en los tres casos. Los mayores valores de iluminancia se obtienen en la fachada noreste donde se alcanzan valores superiores a los 10000 lux, mientras que los menores valores son cercanos a los 3000 lux, obtenidos en la fachada suroeste del caso 6. El caso 4, por su baja relación H/W, presenta valores de iluminancia regulares entre sus niveles de piso. El caso 5 es el que muestra los mayores valores de iluminancia, aunque solamente en su último nivel, alcanzando valores cercanos a los 12000 lux en la fachada noreste y sobre los 8000 lux en la fachada suroeste. El caso 6, al poseer la mayor relación H/W, alcanza los menores valores de iluminancia, los que son de entre 5000 y 10000 lux en la fachada noreste y de valores entre 3000 y 7000 lux en la
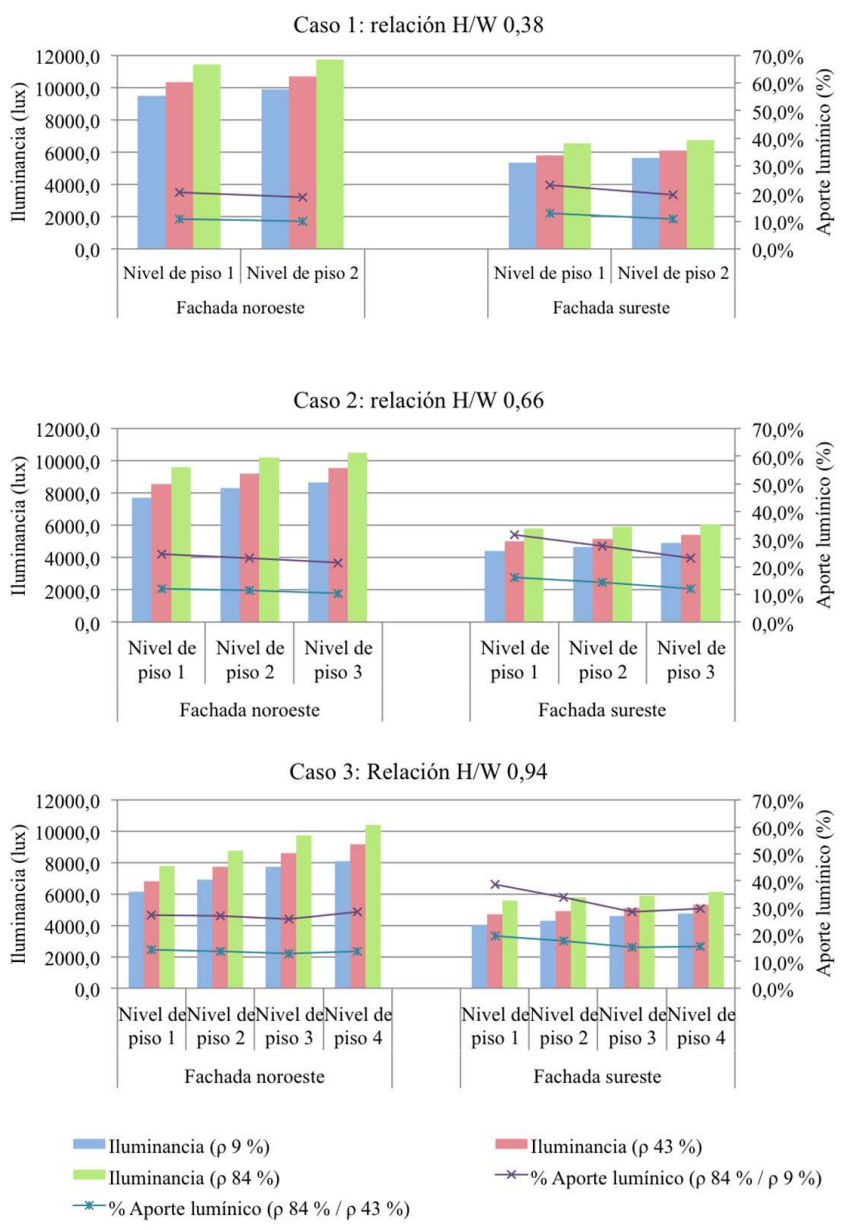

Figura7: Gráficos de iluminancia promedio y aporte lumínico por reflectancia del material de fachada para la orientación 1. Fuente: Elaboración del autor.

fachada suroeste. En la Figura 8, se advierte que las diferencias de iluminancia alcanzadas entre los distintos coeficientes de reflectancia son similares y regulares para todos los casos: de valores entre los 1500 y 2000 lux, comparando las reflectancias de $9 \%$ y $84 \%$. Se dan también diferencias de iluminancia entre los distintos niveles de piso de los casos: los primeros niveles alcanzan los menores valores de iluminancia y los últimos niveles alcanzan los mayores para todos los casos. El caso 4 muestra valores de iluminancia relativamente semejantes entre los niveles de piso de los cañones urbanos, pero en los casos 5 y 6, por sus mayores relaciones H/W, se observa una gran diferencia entre los niveles de ambas fachadas, la cual es de valores cercanos a los 4000 lux, al comparar los primeros y últimos niveles de ambas fachadas.

En cuanto al porcentaje de aporte lumínico por reflectancia del material de fachada, la Figura 8 deja ver que el aporte de luz natural por reflectancia del material es mayor conforme aumenta la diferencia de reflectancias comparadas. Asimismo, puede notarse que el aporte será mayor conforme aumenta la relación $\mathrm{H} / \mathrm{W}$, principalmente en la fachada suroeste, donde se alcanzan aportes de $20 \%$ y $35 \%$ en el caso 4 , de $30 \%$ y 

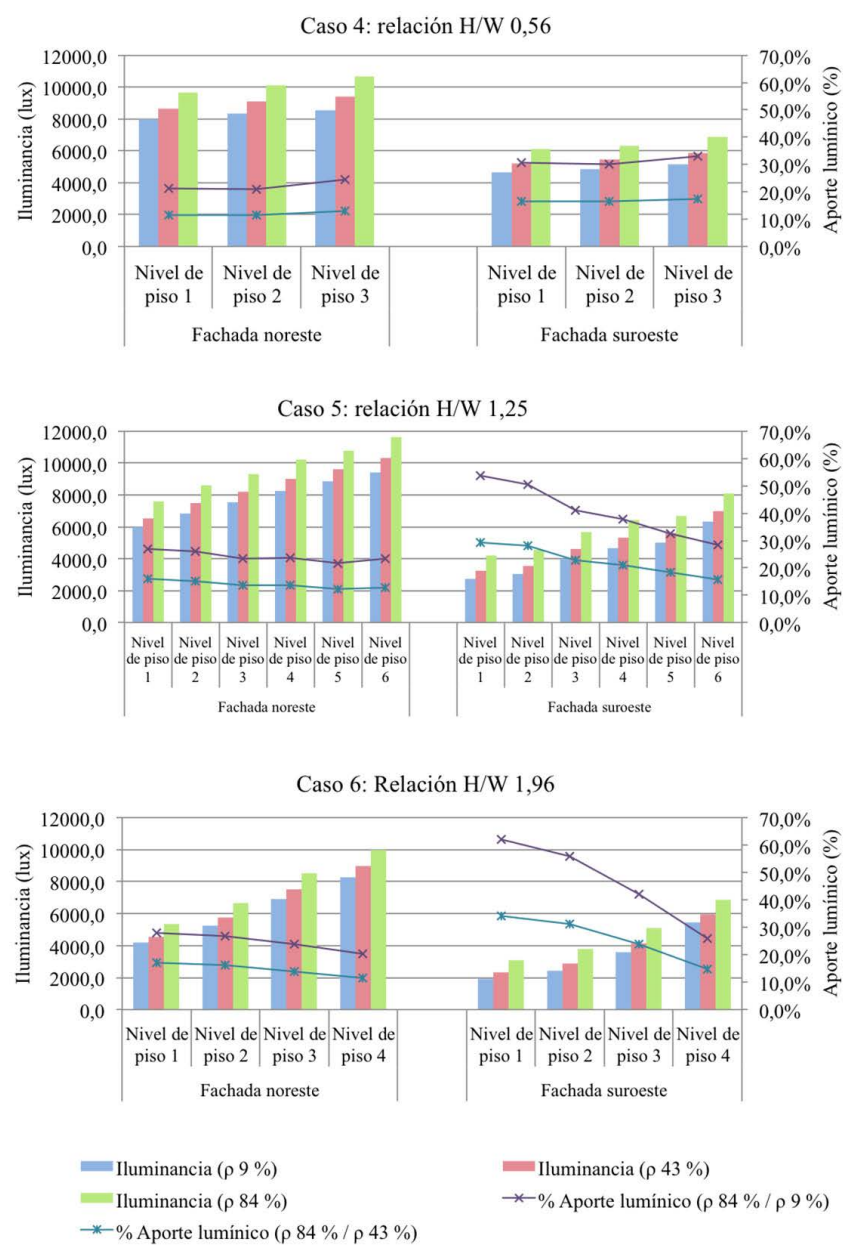

Figura 9: Gráfico de iluminancia y aporte lumínico por reflectancia del material de fachada promedio comparativo.

Fuente: Elaboración del autor.

$55 \%$ en el caso 5 , y de $35 \%$ y $60 \%$ en el caso 6 ; al comparar los coeficientes de reflectancia $84 \%$ / $43 \%$ y $84 \% / 9 \%$, respectivamente.

Según los niveles de piso de los cañones urbanos, se observa que el caso 4 no exhibe grandes diferencias entre sus niveles por su baja relación $\mathrm{H} / \mathrm{W}$, pero en los casos 5 y 6 esta diferencia es muy significativa. En la fachada noreste el porcentaje de aporte se mantiene relativamente constante, mientras que en la fachada suroeste, en los primeros niveles, el aporte de luz natural es mayor para todos los casos, principalmente en los casos 5 y 6 . En el caso 5, los aportes en el primer nivel alcanzan los $35 \%$ y $60 \%$, mientras que en el último nivel son de $15 \%$ y $30 \%$. En el caso 6, los aportes en el primer nivel son de $30 \%$ y $55 \%$, mientras que en el último son de $15 \%$ y $25 \%$, al comparar los valores de reflectancia $84 \%$ / $43 \%$ y $84 \%$ / $9 \%$, respectivamente.

\section{Análisis comparativo cañones urbanos.}

La Figura 9 evidencia un vínculo entre la relación $\mathrm{H} / \mathrm{W}$ de cada cañón y la disponibilidad de luz natural para cada uno de los casos y materiales estudiados, ya que el caso 1, con la

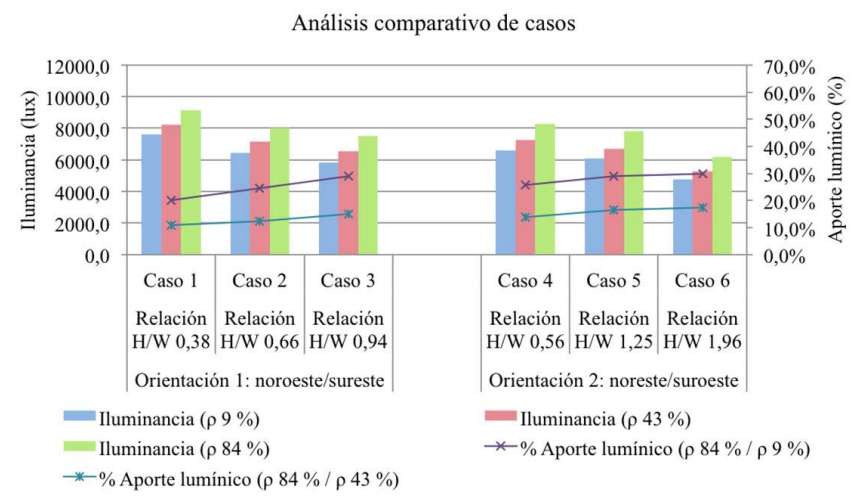

Figura 10: Gráfico de iluminancia y aporte lumínico por reflectancia del material de fachada promedio comparativo. Fuente: Elaboración del autor.

relación H/W más baja, posee los valores de iluminancia más altos entre los casos estudiados, mientras que el caso 6, con la relación $\mathrm{H} / \mathrm{W}$ más alta, presenta los valores más bajos. A pesar de ello, se observa que el aumento de iluminancia debido a la reflectancia del material de fachada es relativamente similar en todos los casos, mostrando diferencias de entre 1500 y 2000 lux, al comparar los materiales de reflectancia $9 \%$ y $84 \%$.

La misma Figura 9 expone un vínculo entre la relación H/W de cada cañón y el porcentaje de aporte lumínico por la reflectancia de los materiales de fachadas. El caso 1, con la relación H/W más baja, es el que en menor porcentaje se ve afectado por el aumento de la reflectancia del material de fachada (Figura 9), mostrando aportes de $10 \%$ y $20 \%$, comparando las reflectancias $84 \%$ / $43 \%$ y $84 \% / 9 \%$, respectivamente. Mientras que el caso 6, con la mayor relación $\mathrm{H} / \mathrm{W}$, presenta el mayor aporte porcentual debido a la reflectancia del material en fachadas entre los casos estudiados, con aportes de 15\% y $30 \%$ en promedio, según las mismas comparaciones. Esto significa que la luz reflejada por la envolvente urbana es la principal fuente de luz diurna aprovechable por los edificios cuando las condiciones geométricas del cañón urbano no permiten obtenerla de forma directa, como en los caso 5 y 6, de modo que puede representar un gran aporte a la disponibilidad de luz natural cuando la reflectancia de los materiales es mayor.

\section{CONCLUSIONES}

Según la relación $\mathrm{H} / \mathrm{W}$, se concluye que la disponibilidad de luz natural exterior se ve directamente condicionada por la relación geométrica del cañón urbano, la cual disminuye conforme aumenta la relación $\mathrm{H} / \mathrm{W}$. El caso 1 , que constituye el cañón urbano de menor relación $H / W(0,38)$, presenta los mayores valores de iluminancia dentro de los casos estudiados, mientras que el caso 6 , con la relación H/W más alta entre los casos estudiados $(1,96)$, presenta los menores valores de iluminancia. Esto ocurre debido a que el cono de sombra generado por la altura de los edificios y el distanciamiento entre sus fachadas limita la ganancia de luz solar directa, disminuyendo la disponibilidad de luz natural aprovechable por los edificios cuando la relación H/W es mayor. 
En el análisis del porcentaje de aporte lumínico, debido a la reflectancia del material de fachada, se demuestra que el aporte será mayor conforme aumenta la relación $\mathrm{H} / \mathrm{W}$, es decir, que en este tipo de cañones urbanos los materiales presentes en las fachadas deberían ser de alta reflectancia para que aporten la mayor cantidad de luz recibida por el sol y traducida en la iluminancia natural exterior. También se comprueba que el aporte de iluminancia será mayor conforme aumenta la diferencia de reflectancia entre los materiales comparados ya que el aporte lumínico entre las reflectancias 84 \% / 9 \% alcanzan los mayores valores de aportes porcentuales conforme aumenta la relación H/W del cañón urbano.

El análisis revela, asimismo, que las fachadas sureste (orientación 1) y suroeste (orientación 2) son las más favorecidas en cuanto al porcentaje de iluminancia aportados por los materiales de fachada. Esto se debe a que las fachadas no se benefician directamente del coeficiente de reflectancia de su propia materialidad, sino que de los materiales de las fachadas y elementos a los cuales se ven enfrentadas, en este caso, las fachadas noroeste (orientación 1) y noreste (orientación 2), que obtuvieron mayores valores de iluminancia en el análisis.

Respecto a los niveles de piso de los edificios, porcentualmente los primeros fueron más beneficiados por el aporte de luz natural debido a la reflectancia de los materiales. Esto se produce ya que los primeros niveles de piso son los que reciben menor luz natural directa, sobre todo en relaciones H/W mayores; razón por la cual la luz natural indirecta obtenida, producto de la reflectancia de los materiales, es la principal fuente de luz diurna en estos niveles. Ahora bien, lo señalado no significa que los primeros niveles sean los únicos a considerar en la selección de los materiales de las fachadas, ya que la luz solar viaja a lo largo de todo el perfil de ésta antes de ser aprovechada por los primeros niveles del cañón urbano.

Se comprueba, además, que la luz reflejada contribuye de manera importante a la disponibilidad de luz natural de los edificios $y$, de hecho, es la mayor parte de la luz diurna disponible para las fachadas menos favorables y pisos más bajos en densidades urbanas altas, cuando la luz natural no puede obtenerse en forma directa; la que, de esta manera, aporta a una distribución más equitativa y homogénea. Es importante considerar que los edificios no son solo estructuras que deben ser valoradas de forma aislada para crear condiciones interiores y exteriores adecuadas, sino que también se debe analizar la influencia de estos sobre el contexto urbano en el que se encuentran inmersos, en aras de asegurar que su desarrollo contribuya a la creación de buenas condiciones de luz diurna para el propio edificio y los edificios vecinos.

Se concluye, en definitiva, que el estudio del cañón urbano, mediante el uso de la relación geométrica $H / W$, es un procedimiento de análisis capaz de reconocer de manera simplificada características esenciales utilizadas en estudios sobre las propiedades de la envolvente y morfología urbana -como densidad, propiedades de los materiales de la envolvente, porcentaje de ventanas, entre otras-, que permite realizar investigaciones de sustentabilidad en cuanto a temperaturas urbanas, vientos, disponibilidad de luz natural y al efecto de las variables del cañón urbano sobre tales factores.

\section{REFERENCIAS BIBLIOGRÁFICAS}

ALCHAPAR, Noelia y CORREA, Érica. Reflectancia solar de las envolventes opacas de la ciudad y su efecto sobre las temperaturas urbanas. Informes de la Construcción, 2015, vol. 67, nº 540, p. 112

ALCHAPAR, Noelia; CORREA, Érica y CANTÓN, María Alicia. Índice de reflectancia solar de revestimientos verticales: potencial para la mitigación de la isla de calor urbana. Ambiente Construido, 2012, vol. $12, n^{\circ} 3$, pp. 107-123

ARIAS, Silvia y ÁVILA, David. La iluminación natural en la arquitectura. México: Editorial Universidad de Guadalajara, 2007.

CÓRICA, Lorena y PATTINI, Andrea. Protocolo de mediciones de iluminación natural en recintos urbanos. Revista Averma, 2005, vol. 9, pp. 05.85-05.90

FREEWAN, Ahmed AY; GHARAIBEH, Anne A. y JAMHAWI, Monther M. Improving daylight performance of light wells in residential buildings: Nourishing compact sustainable urban form. Sustainable Cities and Society, 2014, vol. 13, pp. 32-40.

GAFFIN, S. R., IMHOFF, M., ROSENZWEIG, C., KHANBILVARDI, R., PASQUALINI, A., KONG, A. Y. Y., GRILLO, D., FREED, A., HILLEL, D. y HARTUNG, E. Bright is the new black-multi-year performance of highalbedo roofs in an urban climate. Environmental Research Letters, 2012, vol. 7, n 1, pp. 014029.

GREENUP, Phillip y EDMONDS, I. R. Test room measurements and computer simulations of the micro-light guiding shade daylight redirecting device. Solar Energy, 2004, vol. 76, n 1-3, pp. 99-109.

ILUSTRE MUNICIPALIDAD DE CONCEPCIÓN. Ordenanza local del plan regulador comunal de Concepción [en línea]. [Consultado 20 octubre 2017]. Disponible en: https://www.concepcion.cl/wp-content/ uploads/2013/11/Ordenanza-PRCC-Incluye-modif-Ley-20.791-ydecreto-154-de-2015.pdf

MESA, Néstor Alejandro; CORICA, Lorena y PATTINI, Andrea. Evaluation of the potential of natural light to illuminate buildings in dense urban environment. A study in Mendoza, Argentina. Renewable energy, 2011, vol. 36, nº 9, pp. 2414-2423.

MONTEOLIVA, Juan Manuel y PATTINI, Andrea Elvira. Iluminación natural en aulas: Análisis productivo dinámico del rendimiento lumínicoenergético en clima soleado. Ambiente Construido, 2013, vol. 13, n 4, pp. 235-248.

NASROLLAHI, Nazanin y SHOKRI, Elham. Daylight illuminance in urban environments for visual comfort and energy performance. Renewable and Sustainable Energy Reviews, 2016, vol. 66, pp. 861-874.

PIDERIT, María Beatriz. Ilumina Chile [en línea]. [Consultado 11 septiembre 2017]. Disponible en: http://iluminachile.ubiobio.cl/

RAMÍREZ, David Carlos y OROZCO, Silvia. La envolvente arquitectónica y su influencia en la iluminación natural. Hábitat Sustentable, 2015, vol. $5, n^{\circ} 1$, pp. $44-53$

STRØMANN-ANDERSEN, Jakob y SATTRUP, Peter Andreas. The urban canyon and building energy use: Urban density versus daylight and passive solar gains. Energy and Buildings, 2011, vol. 43, nº 8, pp. 2011 2020.

YUAN, Jihui; FARNHAM, Craig y EMURA, Kazuo. Development of a retro-reflective material as building coating and evaluation on albedo of urban canyons and building heat loads. Energy and Buildings, 2015, vol. 103, pp. 107-117. 